\title{
Tratamento cirúrgico de celulite orbitária e trismo causada por corpo estranho intraorbitário
}

\author{
Surgical treatment of orbitary cellulitis and trismus caused by an intraorbital foreign body \\ Tratamiento quirúrgico de celulitis orbitaria y trismo causado por cuerpo intraorbitario
}

Recebido: 25/03/2021 | Revisado: 31/03/2021 | Aceito: 01/04/2021 | Publicado: 12/04/2021

Priscilla Sarmento Pinto

ORCID: https://orcid.org/0000-0002-2376-4383 Faculdade de Odontologia de Pernambuco, Brasil

Universidade Federal de Pernambuco, Brasil E-mail: priscillasarmentop@gmail.com

Rosa Rayanne Lins de Souza

ORCID: https://orcid.org/0000-0002-9534-605X

Faculdade de Odontologia de Pernambuco, Brasil

E-mail: rosaalins27@hotmail.com

Ruan de Sousa Viana

ORCID: https://orcid.org/0000-0003-1542-4554

Faculdade de Odontologia de Pernambuco, Brasil

E-mail: ruansv@hotmail.com

Demóstenes Alves Diniz

ORCID: https://orcid.org/0000-0001-5823-6364

Hospital da Restauração, Brasil

E-mail: dinizdemostenes@gmail.com

Felipe Ricardo Cisneiros Brito

ORCID: https://orcid.org/0000-0001-8222-9033

Faculdade de Odontologia de Pernambuco, Brasil

E-mail: felipe.cisneiros@upe.br

Dirceu de Oliveira Filho

ORCID: https://orcid.org/0000-0003-2275-966X

Hospital da Restauração, Brasil

E-mail: Dirceudeoliveirafilho@hotmail.com

Greiciane Miguel de Azevedo Santos

ORCID: https://orcid.org/0000-0001-7876-3655

Faculdade de Odontologia de Pernambuco, Brasil

E-mail: greiciane_azevedo@outlook.com

Arthur Alves Thomaz de Aquino

ORCID: https://orcid.org/0000-0002-5664-015X

Faculdade de Odontologia de Pernambuco, Brasil

E-mail: arthur.aquino29@gmail.com

Ronaldo Gabriel Martiniano da Silva

ORCID: https://orcid.org/0000-0001-9248-9277 Centro Universitário Tiradentes de Pernambuco, Brasil

E-mail: Ronaldo.rgms77@gmail.com

Leandro Pimentel Cabral

ORCID: https://orcid.org/0000-0002-4094-1345

Universidade Federal de Pernambuco, Brasil

E-mail: Cabral.leop@gmail.com

\begin{abstract}
Resumo
Desenvolver uma revisão integrativa acerca da infecção orbital por corpo estranho penetrante, através da análise do sexo e idade mais prevalente, etiologia, sinais e sintomas, abordagem cirúrgica e sequelas. Além disso, relatar um caso de infecção orbitária por um corpo estranho penetrante com severa limitação de abertura bucal. Consiste em duas etapas, na primeira, dois autores procuraram independentemente artigos sobre infecção orbital por corpo estranho penetrante nas bases de dados "MEDLINE/PubMed", "Scielo" e "Scopus", utilizando descritores combinados com os operadores booleanos. A segunda etapa consiste em um relato de caso de celulite orbitária após penetração de corpo estranho ("pedaço" de madeira) em assoalho de órbita. Essa lesão se mostrou mais comum no sexo masculino (75\%), com idade média de 41,7 anos, decorrente do acidente de trabalho $(33,3)$, evoluindo com sinais e sintomas de lesão cutânea, dor, oftalmoplegia, baixa acuidade visual e abscesso. Sendo a abordagem cirúrgica mais usada a orbitotomia $(41,7 \%)$, com os pacientes sem sequelas pós-cirúrgicas $(50 \%)$. A condução do caso deve ser por uma equipe multidisciplinar, com abordagem imediata, evitando assim disseminação da infecção para região intracraniana. Concluiu-se também que o risco de infecção está diretamente relacionado a natureza do material do corpo estranho.
\end{abstract}


Palavras-chave: Órbita; Trismo; Corpos estranhos no olho.

\begin{abstract}
Develop an integrative review about orbital infection by penetrating foreign body, through the analysis of gender and most prevalent age, etiology, signs and symptoms, surgical approach and sequelae. In addition, to report a case of orbital infection by a penetrating foreign body with severe mouth opening limitation. It consists of two stages: first, two authors independently searched for articles on orbital infection by penetrating foreign bodies in the databases "MEDLINE / PubMed", "Scielo" and "Scopus ", using descriptors combined with the Boolean operators. The second stage consists of a case report of orbital cellulitis after penetration of a foreign body ("piece" of wood) in an orbit floor. This lesion was more common in males (75\%), with an average age of 41.7 years, due to work accidents (33.3), evolving with signs and symptoms of skin lesion, pain, ophthalmoplegia, low visual acuity and abscess. The most used surgical approach is the orbitotomy $(41.7 \%)$, with patients without post-surgical sequelae $(50 \%)$. The case should be managed by a multidisciplinary team, with an immediate approach, avoiding the spread of infection to the intracranial region. It was also concluded that the risk of infection is directly related to the nature of the foreign body material.
\end{abstract}

Keywords: Orbit; Trismus; Eye foreign bodies.

\title{
Resumen
}

Desarrollar una revisión integradora sobre la infección orbitaria por penetración de cuerpo extraño, mediante el análisis del sexo y edad más prevalente, etiología, signos y síntomas, abordaje quirúrgico y secuelas. Además, reportar un caso de infección orbitaria por cuerpo extraño penetrante con severa limitación de la apertura bucal. Consta de dos etapas, en la primera, dos autores buscaron de forma independiente artículos sobre infección orbitaria por penetración de cuerpos extraños en las bases de datos "MEDLINE / PubMed", "Scielo" y "Scopus", utilizando descriptores combinados con los operadores booleanos. La segunda etapa consiste en el reporte de un caso de celulitis orbitaria luego de la penetración de un cuerpo extraño ("pieza" de madera) en un piso orbital. Esta lesión fue más frecuente en el sexo masculino (75\%), con una edad media de 41,7 años, por accidente laboral $(33,3)$, evolucionando con signos y síntomas de lesión cutánea, dolor, oftalmoplejía, baja agudeza visual. y absceso. El abordaje quirúrgico más utilizado es la orbitotomía (41,7\%), con pacientes sin secuelas posquirúrgicas (50\%). El caso debe ser manejado por un equipo multidisciplinario, con abordaje inmediato, evitando así la propagación de la infección a la región intracraneal. También se concluyó que el riesgo de infección está directamente relacionado con la naturaleza del material del cuerpo extraño.

Palabras clave: Órbita; Trismo; Corps étrangers oculaires.

\section{Introdução}

Lesões de alta velocidade podem levar à ocorrência de um corpo estranho intraorbitário (Moraes et al., 2015), essa lesão ocular é incomum e ocorre com uma frequência aproximadamente de 16\% de todas as lesões orbitais (Chen et al., 2015; Cho et al., 2017; Mirzaei et al., 2019; Tabibkhooei et al., 2019; Mohapatra et al., 2020). O corpo estranho intraorbitário é geralmente relacionado a acidentes envolvendo trauma penetrante de alta velocidade (Moraes et al., 2015; Blackhall and Laraway., 2016 Mirzaei et al., 2019; Szabo et al., 2019) e tipicamente acometem homens, crianças e adultos jovens (Mirzaei et al., 2019; Szabo et al., 2019). A gravidade do trauma é avaliada em relação à velocidade e trajetória do objeto, normalmente causada por quedas, agressões ou colisões de veículos a motor (Tabibkhooei et al., 2019).

Um corpo estranho intraorbitário é definido como um objeto localizado dentro das paredes ósseas orbitais, sendo posterior ao septo orbital, porém fora do globo ocular. Em relação a sua posição, pode ser classificada como extracornal ou intracornal, já a sua natureza, pode ser metálica ou não metálica, sendo estes, orgânicos ou não orgânicos (Szabo et al., 2019). Como manejo geral, antibióticos empíricos de amplo espectro devem ser administrados, principalmente quando falamos dos casos envolvendo corpo estranho de madeira, em razão de sua natureza torná-la um reservatório ideal para bactérias (JusuéTorres et al., 2016; Li et al., 2016; Cho et al., 2017; Szabo et al., 2019).

A órbita está no limite do neurocrânio e viscerocrânio, dessa forma devido às particularidades anatômicas, os corpos estranhos intraorbitais podem se estender para o interior do crânio (Singh et al., 2018), levando um risco de não apenas prejudicar a visão, mas também de infecção ao cérebro (Chen et al., 2015). Além disso, por causa das delgadas paredes ósseas órbitais, um acesso é provido para a cavidade craniana e isso pode resultar em danos severos aos olhos, nervos ópticos, cérebro 
e vasculatura cerebral (Tabibkhooei et al., 2019). Assim, no contexto de trauma orbital a necessidade de um manejo multidisciplinar torna-se imprescindível (Szabo et al., 2019).

Dentro do contexto das infecções orbitais, é válido abordar os seus tipos: Celulite Pré-septal, a qual as estruturas posteriores ao septo orbital não estão infeccionadas, porém, podem estar inflamadas; Celulite Orbital que implica em infecção ativa do tecido mole retro septal da órbita e o tecido mole pré-septal pode estar infeccionado ou inflamado; Abscesso Orbital; Infecções Orbitais Fúngicas Invasivas (Topazian et al., 2006).

Portanto, o propósito desse estudo foi desenvolver uma revisão integrativa sobre infecção orbital por corpo estranho penetrante, através da análise do sexo, idade, etiologia, sinais e sintomas, abordagem cirúrgica e sequelas mais prevalentes. Além disso, relatar um caso clínico de infecção orbitária e trismo relevante, causados por objeto estranho intraorbitário penetrante.

\section{Metodologia}

\section{Desenho do estudo, problemas dos pacientes e revisão integrativa}

Este estudo foi realizado em duas etapas de acordo com o método previamente descrito (Whittemore and Knafl., 2005; Oliveira et al., 2018). Na primeira etapa, dois autores (PSP e RSV) procuraram independentemente em abril de 2020, artigos sobre infecção orbital por corpo estranho penetrante nas seguintes bases de dados "MEDLINE/PubMed", "Scielo" e "Scopus". Para esta busca foram utilizados os seguintes descritores combinados com os operadores booleanos: (cellulitis OR abcesso or suppuration) AND (maxillofacial injuries OR orbital injury OR facial injuries OR eye foreign bodies OR eye injuries OR eye injuries penetrating) limitados aos últimos 10 anos. Os critérios de inclusão para seleção de artigos foram relato de casos, série de casos, estudos prospectivos ou retrospectivos, full text availability in English, Portuguse or Spanish, artigos que reportem infecção orbitária pela presença de corpo estranho penetrante.

No segundo momento, é relatado caso de celulite orbitária após penetração de corpo estranho ("pedaço" de madeira) em assoalho de órbita. O paciente foi informado sobre a publicação do caso e assinou o termo de consentimento livre e esclarecido.

Os seguintes dados foram extraídos de cada estudo quando presentes: autor, ano de publicação, sexo e idade, etiologia do trauma, sinais e sintomas, e sequelas pós-cirúrgicas.

Os artigos foram selecionados inicialmente pelos títulos, refinado pelos resumos. Após leitura dos resumos, os estudos que pareceram corresponder ao objetivo desta revisão, foram lidos integralmente, e uma vez que preencheram os critérios de inclusão, participaram deste estudo.

\section{Análise de Dados}

O Software SPSS (version 23.0; IBM, Armonk, NY) foi usado para estatística descritiva dos dados sociodemográficos e dos casos de infecção orbital por corpo estranho penetrante da literatura.

\section{Considerações Éticas}

Os dados do paciente em questão foram coletados por meio do acesso direto ao prontuário e exames solicitados. Seguindo os princípios da ética, o paciente consentiu com a divulgação de seu caso com finalidade acadêmica por meio da assinatura de um Termo de Consentimento Livre e Esclarecido. Todos os princípios éticos descritos por Helsinque foram respeitados, objetivando proteger a vida, saúde, privacidade e dignidade do ser humano. Os dados do paciente foram avaliados após diagnóstico. 


\section{Relato De Caso}

Paciente gênero masculino, 29 anos de idade, foi atendido no Hospital da Restauração Governador Paulo GuerraRecife/PE, o mesmo veio encaminhado de centro oftalmológico da cidade para avaliação Buco-Maxilo-Facial, com história de acidente motociclístico há aproximadamente 02 meses, evoluindo com celulite periorbitária em olho direito e limitação de abertura bucal após o trauma. Ao exame físico apresentava-se com proptose ocular em olho direito, associada à saída de secreção purulenta, e fístula cutânea infra-orbital, bem como queixa de baixa acuidade visual em referido olho, restrição dos movimentos oculares e severa limitação de abertura bucal (10 mm de abertura máxima).

Figura 1 - a) Observa-se a proptose ocular em olho direito, associada à saída de secreção purulenta e fístula cutânea infraorbital; b) Limitação severa da abertura bucal, associada ao corpo estranho infraorbital.

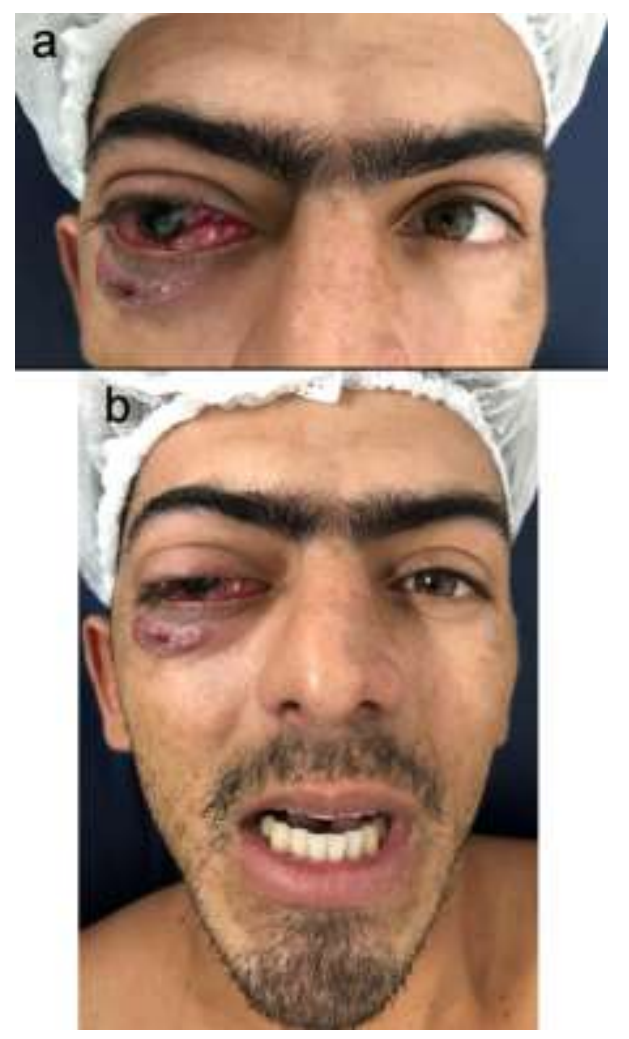

Fonte: Autores.

Ao exame de imagem (Tomografia Computadorizada de Face) observou-se presença de corpo estranho transfixando de assoalho orbital estendendo-se até processo coronóide mandibular ipsilateral, além disso, apresentava leucocitose e febre. 
Figura 2 - a) TC 3D, presença de corpo estranho transfixado do assoalho da órbita (círculo); b) TC 3D, o corpo estranho se estende para o processo coronoide mandibular ipsilateral, causando limitação severa da abertura bucal (círculo); c) TC axial, corpo estranho infraorbital (círculo).

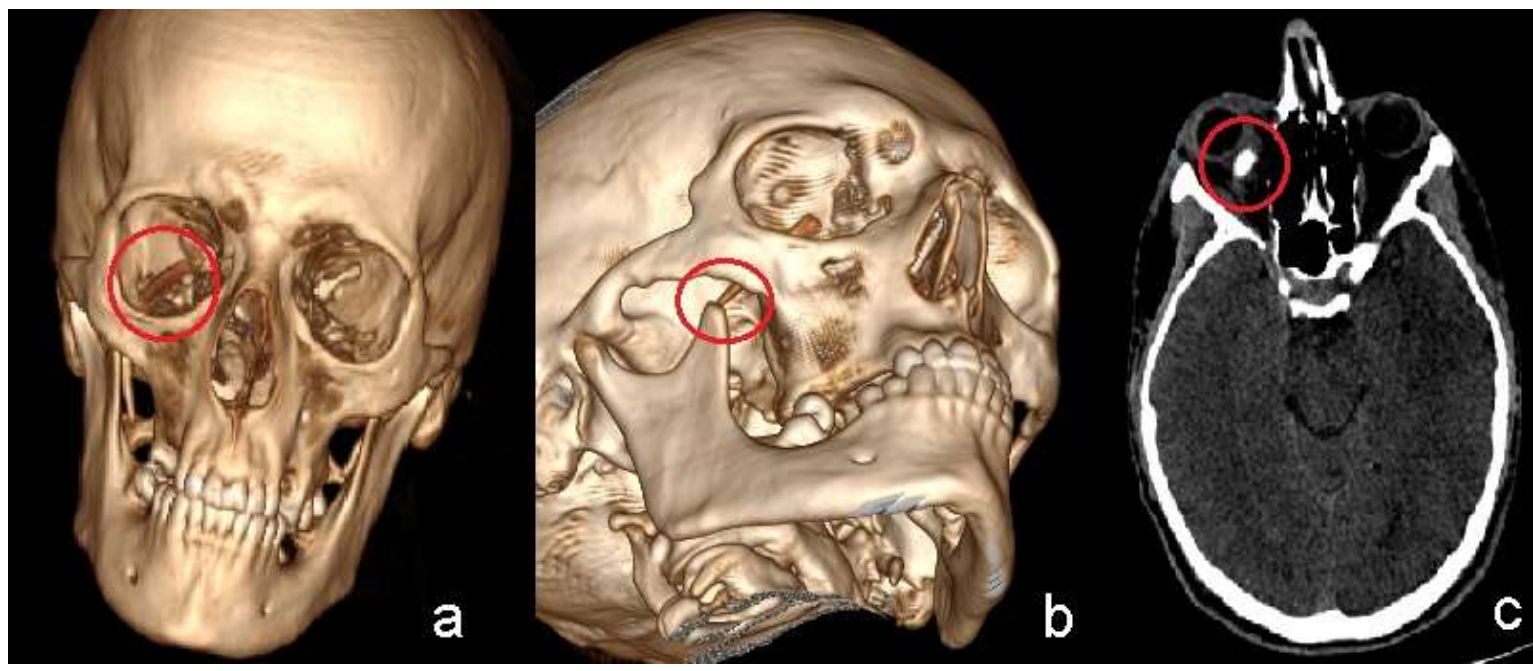

Fonte: Autores.

Foi instituído imediatamente uso de ceftriaxona (1g IV 12/12 h) e oxacilina (1g IV 6/6 h), e então paciente foi submetido à cirurgia sob anestesia geral para remoção de corpo estranho (madeira), fistulectomia e limpeza cirúrgica, pela equipe CTBMF do serviço com acompanhamento do oftalmologista de plantão.

Figura 3 - a) Remoção de corpo estranho por acesso infraorbital. Tarsorrafia foi realizada; b) Fistulectomia e limpeza cirúrgica; c) Sutura do acesso infraorbital; d) Remoção total do corpo estranho infraorbital.

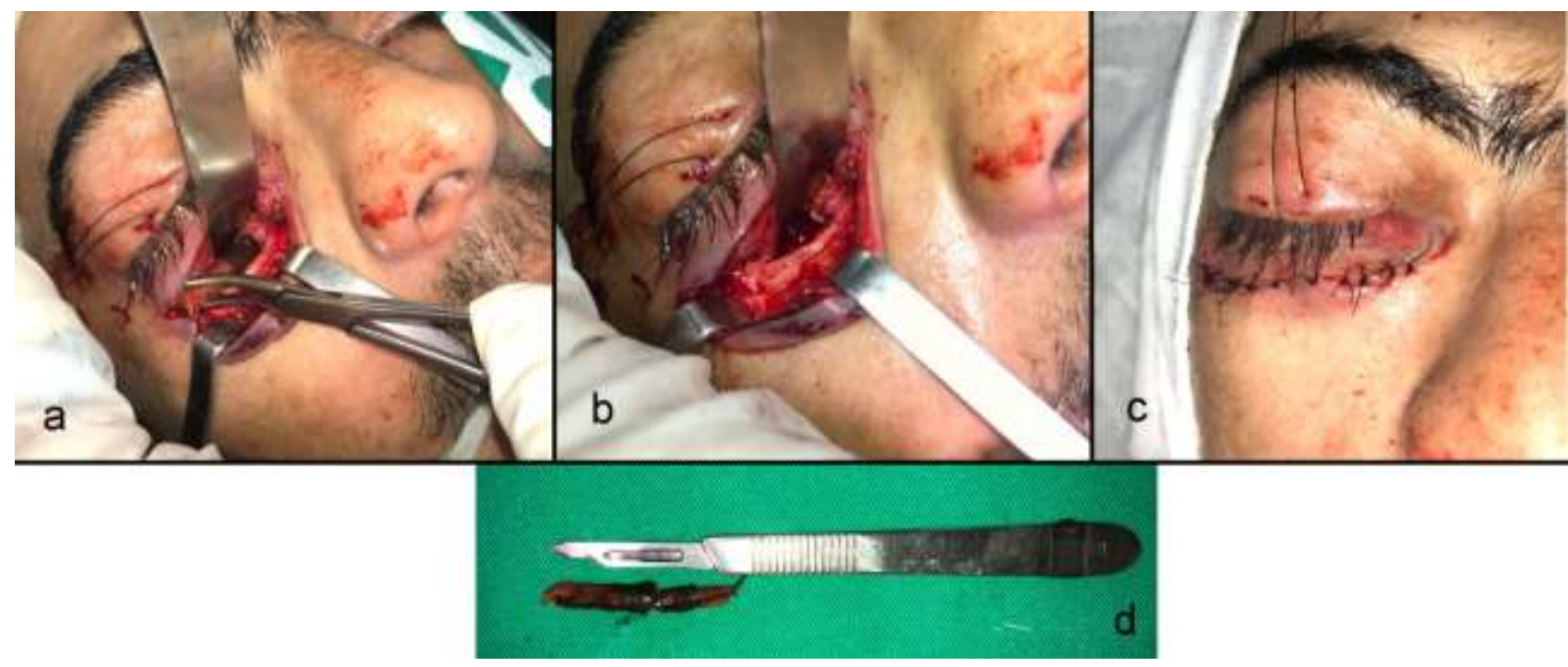

Fonte: Autores.

A antibioticoterapia endovenosa foi mantida por 07 dias e prescrito antibioticoterapia via enteral para casa com Amoxicilina 875mg + Ácido Clavulânico 125mg 8/8 horas por 07 dias. O mesmo segue em acompanhamento ambulatorial (CTBMF, oftalmologista e fisioterapia), após 03 meses, com melhora da visão, movimentos oculares preservados, e melhor abertura bucal (25mm de abertura máxima). 
Figura 4 - a) TC 3D, pós-operatório; b) TC 3D, pós-operatório. É visualizada a remoção total do corpo infra-orbital, cessando o impacto do processo coronóide mandibular; c) TC axial, sem a presença de corpo estranho infraorbital.

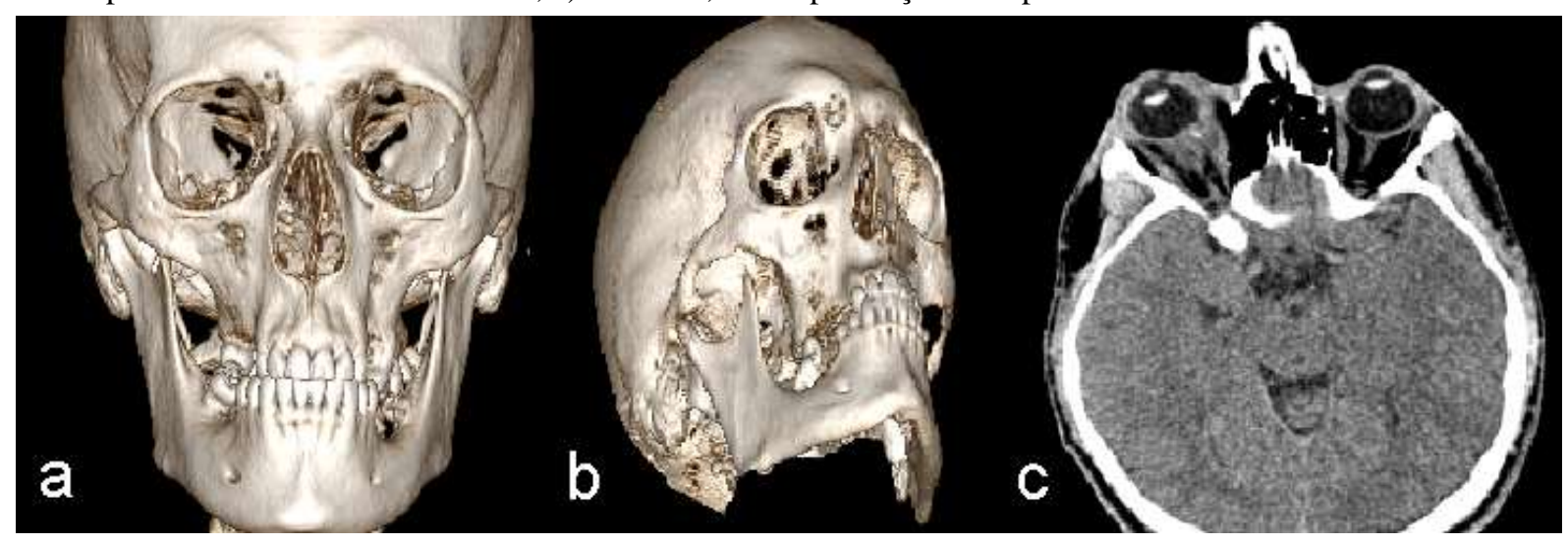

Fonte: Autores.

Figura 5 - a) Abertura máxima da boca até $25 \mathrm{~mm}$, após 03 meses; b) Mobilidade ocular após a cirurgia, após 03 meses. Diferença entre o olho esquerdo e o direito; c) Mobilidade ocular para superversão após 3 meses de cirurgia; d) Mobilidade ocular e lateroversão após 3 meses da cirurgia.

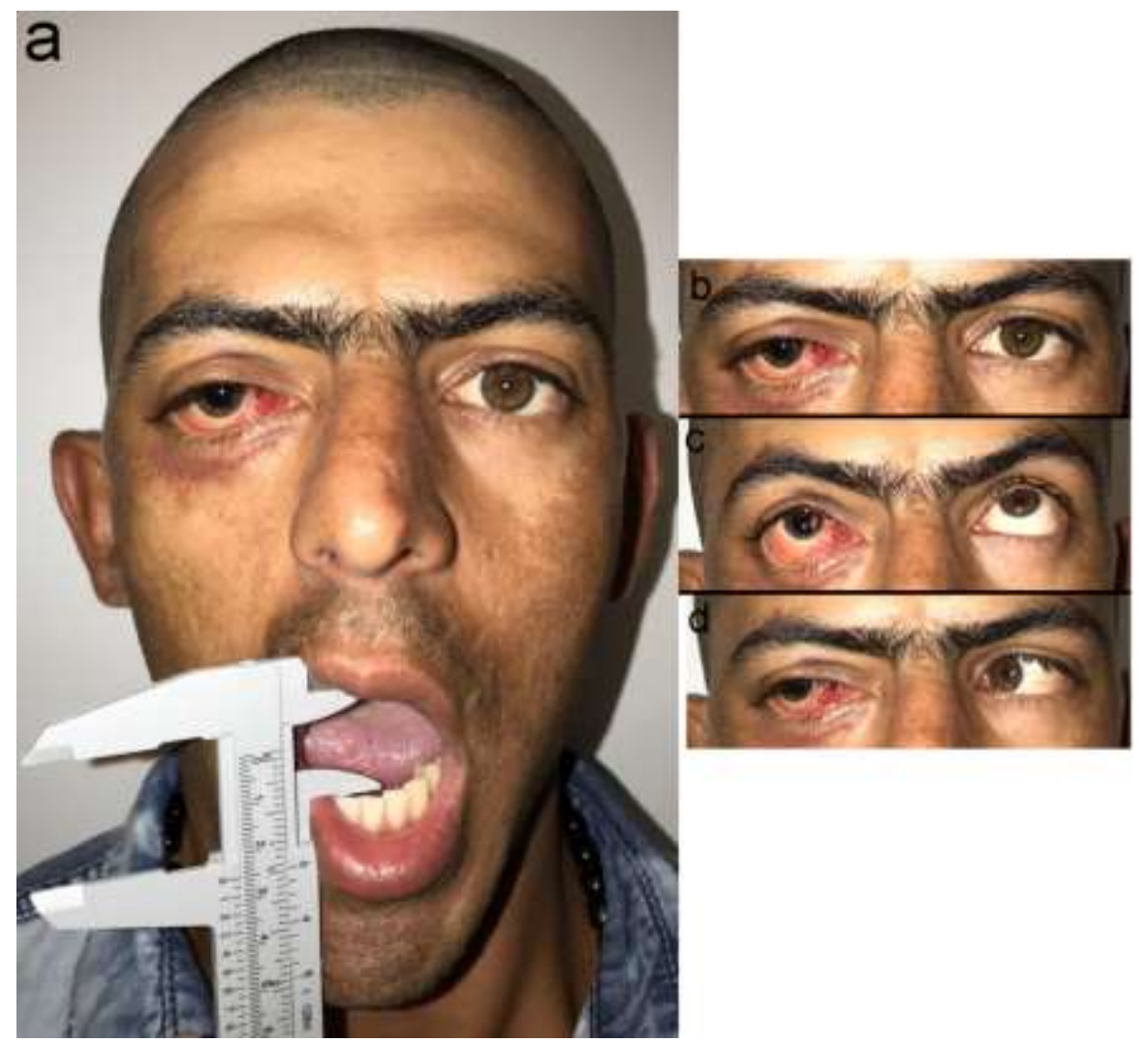

Fonte: Autores.

\section{Resultados}

\section{Revisão Integrativa}

A revisão integrativa produziu 319 artigos. Após leitura dos títulos foram selecionados 19 artigos, sendo que 02 não possuíam o manuscrito na íntegra para leitura e avaliação; 01 se tratava de um pino de uma prótese que adentrou na órbita; outro era referente a celulites orbitárias não provocadas por corpo estranho; 01 referia-se a penetração e abscesso intracraniano; outro tratava-se de aneurisma de carótida por trauma penetrante intraorbital. Seguindo com a leitura dos resumos foram 
selecionados 13 artigos, sendo 11 artigos de relatos de casos, o qual um deles apresentou 02 casos relatados e 02 estudos retrospectivos (Tabela suplementar 1).

Tabela suplementar 1 - Detalhamento dos artigos encontrados na literatura sobre celulite orbitária por corpo estranho.

\begin{tabular}{|c|c|c|c|c|c|c|}
\hline $\begin{array}{l}\text { Autor/ ano de } \\
\text { publicação }\end{array}$ & Sexo & Idade & $\begin{array}{l}\text { Etiologia do } \\
\text { trauma }\end{array}$ & Sinais e sintomas & Aborgem cirurgica & Sequelas \\
\hline $\begin{array}{l}\text { Rajendran and } \\
\text { Rikhotso (2019) }\end{array}$ & $\mathrm{F}$ & 25 & $\begin{array}{l}\text { Agressão } \\
\text { física } \\
\text { (FACA); }\end{array}$ & $\begin{array}{l}\text { 1-Oftalmoplegia; } \\
\text { 2-Laceração intra-orbital; }\end{array}$ & $\begin{array}{l}\text { Transcutânea pelo } \\
\text { ferimento } \\
\text { (infraorbitário) }\end{array}$ & $\begin{array}{l}\text { Paciente não } \\
\text { apresentou sequelas; }\end{array}$ \\
\hline $\begin{array}{l}\text { Bayramoğlu et al } \\
\text { (2018) }\end{array}$ & $\mathrm{F}$ & 35 & $\begin{array}{l}\text { Acidente de } \\
\text { Trabalho; }\end{array}$ & $\begin{array}{l}\text { 1-Edema em pálpebra superior } \\
\text { 2-Dor em pálpebra superior; }\end{array}$ & $\begin{array}{l}\text { Orbitotomia } \\
\text { superonasal; }\end{array}$ & Ptose residual; \\
\hline $\begin{array}{l}\text { Kaplan et al } \\
(2018)\end{array}$ & M & 03 & $\begin{array}{l}\text { Acidente de } \\
\text { trenó; }\end{array}$ & $\begin{array}{l}\text { 1-Edema e eritema peri- } \\
\text { orbital, } \\
\text { 2- Ferimento com crosta em } \\
\text { pálpebra inferior. }\end{array}$ & Orbitotomia anterior; & $\begin{array}{l}\text { Paciente não } \\
\text { apresentou sequelas; }\end{array}$ \\
\hline $\begin{array}{l}\text { Ghadersohi et al } \\
\text { (2017) }\end{array}$ & $\mathrm{F}$ & 53 & $\begin{array}{l}\text { Queda de nível } \\
\text { com } \\
\text { penetração de } \\
\text { galho em } \\
\text { órbita; }\end{array}$ & $\begin{array}{l}\text { 1-Cefaléia } \\
\text { 2- Dor em olho } \\
\text { 3- Avulsão de parte de } \\
\text { pálpebra superior e } \\
\text { sobrancelha; }\end{array}$ & $\begin{array}{l}\text { Abordagem } \\
\text { endoscópica e } \\
\text { transorbital } \\
\text { combinada para } \\
\text { remoção corpo } \\
\text { estranho; } \\
\text { Septoplastia, } \\
\text { antrostomia maxilar, } \\
\text { etmoidectomia total, } \\
\text { esfeinodotomia e } \\
\text { sinusotomia frontal; }\end{array}$ & $\begin{array}{l}\text { Paciente não } \\
\text { apresentou sequelas; }\end{array}$ \\
\hline $\begin{array}{l}\text { Miller and Coan } \\
\text { (2016) }\end{array}$ & M & 30 & $\begin{array}{l}\text { Mergulho, } \\
\text { trauma por } \\
\text { peixe agulha; }\end{array}$ & $\begin{array}{l}\text { 1-Dor } \\
\text { 2- Baixa acuidade visual } \\
\text { progressiva } \\
\text { 3-Oftalmoplegia em olho } \\
\text { direito. }\end{array}$ & Orbitotomia Lateral; & $\begin{array}{l}\text { Diplopia em visão } \\
\text { Periférica; }\end{array}$ \\
\hline $\begin{array}{l}\text { Swathi and } \\
\text { Umadevi (2014) }\end{array}$ & $\mathrm{M}$ & 25 & $\begin{array}{l}\text { Acidente de } \\
\text { Trabalho; }\end{array}$ & $\begin{array}{l}\text { 1-Dor ocular; } \\
\text { 2- Baixa acuidade visual; } \\
\text { 3-Oftalmoplegia } \\
\text { 4-proptose; }\end{array}$ & $\begin{array}{l}\text { Endoscopia via } \\
\text { transnasal/transetmoid } \\
\text { al; }\end{array}$ & Baixa acuidade Visual; \\
\hline $\begin{array}{l}\text { Kim and } \\
\text { Sivaramam (2013) } \\
\text { (CASO 1) }\end{array}$ & $\mathrm{M}$ & 6 & Mergulho & $\begin{array}{l}\text { 1-Celulite Orbital; } \\
\text { 2-Edema Palpebral; } \\
\text { 3-Proptose; }\end{array}$ & $\begin{array}{l}\text { Orbitotomina } \\
\text { Superomedial; }\end{array}$ & $\begin{array}{l}\text { Baixa Acuidade } \\
\text { Visual; }\end{array}$ \\
\hline $\begin{array}{l}\text { Kim and } \\
\text { Sivaramam (2013) } \\
\text { (CASO 2) }\end{array}$ & M & 12 & Mergulho & $\begin{array}{l}\text { 1-Febre; } \\
\text { 2-Dor; } \\
\text { 3-Edema Periorbitario, } \\
\text { Proptose; } \\
\text { 4-Vômito; } \\
\text { 5-Celulite orbital; } \\
\text { 6-Baixa acuidade visual; } \\
\text { 7-Diplopia Binocular; }\end{array}$ & $\begin{array}{l}\text { Orbitotomina } \\
\text { Inferomedial; }\end{array}$ & $\begin{array}{l}\text { Baixa Acuidade } \\
\text { Visual; }\end{array}$ \\
\hline Czyz, et al (2012) & $\mathrm{F}$ & 39 & $\begin{array}{l}\text { Acidente de } \\
\text { Trabalho; }\end{array}$ & $\begin{array}{l}\text { 1-Edema periorbitario; } \\
\text { 2-Dor em orbita; } \\
\text { 3-Diminuição progressiva da }\end{array}$ & $\begin{array}{l}\text { Transcutânea pelo } \\
\text { ferimento, (margem } \\
\text { medial de órbita); }\end{array}$ & $\begin{array}{l}\text { 1-Ausência de } \\
\text { percepção luminosa e } \\
\text { B.A.V; }\end{array}$ \\
\hline
\end{tabular}


Research, Society and Development, v. 10, n. 4, e32310414280, 2021

(CC BY 4.0) | ISSN 2525-3409 | DOI: http://dx.doi.org/10.33448/rsd-v10i4.14280

\begin{tabular}{|c|c|c|c|c|c|c|}
\hline & & & & $\begin{array}{l}\text { acuidade visual; } \\
\text { 4-Oftalmoplegia em todas } \\
\text { direções; }\end{array}$ & & \\
\hline Kang et al (2012) & M & 43 & $\begin{array}{l}\text { Agressão por } \\
\text { caneta } \\
\text { esfereográfica; }\end{array}$ & $\begin{array}{l}\text { 1-Oftalmoplegia em todas as } \\
\text { direções; } \\
\text { 2-Dor; }\end{array}$ & $\begin{array}{l}\text { Orbitotomia } \\
\text { infraorbital + } \\
\text { Endoscopia maxilar; }\end{array}$ & $\begin{array}{l}\text { Paciente não } \\
\text { apresentou sequelas; }\end{array}$ \\
\hline $\begin{array}{l}\text { Moretti et al } \\
\text { (2012) }\end{array}$ & M & 50 & $\begin{array}{l}\text { Acidente de } \\
\text { trabalho; }\end{array}$ & $\begin{array}{l}\text { 1-Laceração em região lateral } \\
\text { de pálpebra ; }\end{array}$ & $\begin{array}{l}\text { Transcutânea pelo } \\
\text { ferimento (Palpebra } \\
\text { superior Direita); }\end{array}$ & $\begin{array}{l}\text { Paciente não } \\
\text { apresentou sequelas; }\end{array}$ \\
\hline Yang et al (2010) & M & 56 & $\begin{array}{l}\text { Trauma em } \\
\text { face por } \\
\text { bomba } \\
\text { (explosão); }\end{array}$ & $\begin{array}{l}\text { 1-Cefaleia; } \\
\text { 2- Dor Peri orbital, } \\
\text { 3-Náuseas, vômitos, dispneia, } \\
\text { palidez, sudorese. }\end{array}$ & $\begin{array}{l}\text { Abordagem } \\
\text { intraocular; }\end{array}$ & $\begin{array}{l}\text { Paciente não } \\
\text { apresentou sequelas; }\end{array}$ \\
\hline Autor/ano & $\begin{array}{l}\text { Sexo } \\
\text { preval } \\
\text { ente }\end{array}$ & $\begin{array}{l}\text { Média } \\
\text { de } \\
\text { idade }\end{array}$ & $\begin{array}{l}\text { Etiologia } \\
\text { mais } \\
\text { prevalente }\end{array}$ & $\begin{array}{l}\text { Sinais e sintomas mais } \\
\text { prevalentes }\end{array}$ & $\begin{array}{l}\text { Abordagem } \\
\text { cirúrgica }\end{array}$ & $\begin{array}{l}\text { Sequelas mais } \\
\text { comuns }\end{array}$ \\
\hline Bilge et al (2016) & $\begin{array}{l}M=20 \\
F=4\end{array}$ & $\begin{array}{l}\text { MÉDI } \\
\text { A: } 28 \\
\text { ANOS }\end{array}$ & NR & $\begin{array}{l}\text { Celulite orbital ( } 5 \text { casos) e } \\
\text { neuropatia ótica em } 03 \text { casos; }\end{array}$ & NR & $\begin{array}{l}03 \text { pacientes que } \\
\text { apresentaram } \\
\text { neuropatia ótica } \\
\text { continuaram com } \\
\text { problemas visuais. }\end{array}$ \\
\hline LI et al (2016) & $\begin{array}{l}M=11 \\
F=3\end{array}$ & $\begin{array}{l}36 \\
\text { anos }\end{array}$ & $\begin{array}{l}\text { Lesão por } \\
\text { escorregament } \\
\text { o }\end{array}$ & $\begin{array}{l}\text { 1-Baixa acuidade visual; } \\
\text { 2-Oftalmoplegia; }\end{array}$ & $\begin{array}{l}08 \text { casos por } \\
\text { ferimento, duas } \\
\text { através de lesão } \\
\text { conjutival e } 04 \text { por } \\
\text { orbitotomia anterior; }\end{array}$ & Perda visual \\
\hline
\end{tabular}

Abreviações: M (masculino), F (feminino); NR (não relatado); Nota de rodapé: foram considerados acidentes por instrumental pontiagudo: trauma por faca e trauma por caneta esferográfica. Fonte: Autores.

\section{Amostra}

A amostra (Tabela 1) foi composta por 09 pacientes (75\%) do sexo masculino e 03 do gênero feminino (25\%), quando avaliado os relatos de casos da literatura. Já nos estudos retrospectivos o gênero mais prevalente foi o sexo masculino.

A idade média dos pacientes foi de 31,4 anos (variação de 03 a 56 anos) nos relatos de casos. Nos estudos retrospectivos a média de idade em um dos estudos foi de 28 anos e no outro de 36 anos.

\section{Etiologia}

A maioria dos pacientes estiveram envolvidos em acidentes de trabalho $(\mathrm{n}=4 ; 33 \%)$, seguido por acidente por mergulho $(\mathrm{n}=3 ; 25 \%)$, nos relatos de casos (Tabela 1). Já nos estudos retrospectivos 01 estudo relatou que a etiologia mais prevalente foi acidente por escorregamento e o outro não detalhou essa informação.

\section{Sinais e Sintomas}

Oftalmoplegia isolada esteve presente em 2 casos (16,7\%), 2 outros casos apresentaram oftalmoplegia associados a baixa acuidade visual (BAV) e dor, e ainda 2 casos apresentaram formação de abcesso (16,7\%), quando avaliados os relatos de casos da literatura (Tabela 1). 
Nos estudos retrospectivos um deles apresentou oftalmoplegia associado a BAV como sinais e sintomas mais prevalentes e outra celulite orbitária associada a neuropatia ótica.

\section{Abordagem Cirúrgica}

$\mathrm{Na}$ maioria dos casos foi utilizada a orbitotomia como abordagem cirúrgica $(n=5 ; 41,7 \%)$, seguido de acesso pelo ferimento pré-existente $(\mathrm{n}=3 ; 25 \%)$ nos relatos de casos (Tabela 1$)$.

Em um dos estudos retrospectivos não foi relatado a abordagem cirúrgica mais prevalente, no outro, 10 casos foram abordados pelo ferimento pré-existente, e 04 através de orbitotomia.

\section{Sequelas}

A maioria dos casos (Tabela 1) não apresentaram sequelas após abordagem cirúrgica ( $\mathrm{n}=6 ; 50 \%), 03$ casos apresentaram baixa acuidade visual (25\%).

Um estudo retrospectivo observou-se que dos 24 pacientes avaliados, 03 que apresentaram neuropatia ótica não obteve melhora após abordagem cirúrgica. O outro estudo dos 14 pacientes avaliados, 08 seguiram com perda visual.

\begin{tabular}{|c|c|}
\hline Tabela 1 - INFECÇÕES ORBITÁRIAS P & $\begin{array}{l}\text { ANHO PENETRANTES PUBLICADOS NA LITERATURA } \\
\text { E CASOS }\end{array}$ \\
\hline VARIÁVEL & DADOS \\
\hline \multicolumn{2}{|l|}{ Gênero n (\%) } \\
\hline Masculino & $9(75 \%)$ \\
\hline Feminino & $3(25 \%)$ \\
\hline \multicolumn{2}{|l|}{ IDADE, média (mínimo-máximo) } \\
\hline \multicolumn{2}{|l|}{ ETIOLOGIA DO TRAUMA n (\%) } \\
\hline Acidente de trabalho & $4(33,3 \%)$ \\
\hline Mergulho & $3(25 \%)$ \\
\hline Agressão por objeto pontiagudo & $2(16,7 \%)$ \\
\hline Acidente de Trenó & $1(8,3 \%)$ \\
\hline Queda de nível & $1(8,3 \%)$ \\
\hline Explosão & $1(8,3 \%)$ \\
\hline \multicolumn{2}{|l|}{ SINAIS E SINTOMAS n (\%) } \\
\hline Oftalmoplegia & $2(16,7 \%)$ \\
\hline Lesão Cutânea & $2(16,7 \%)$ \\
\hline Abcesso & $2(16,7 \%)$ \\
\hline Oftalmoplegia + dor + BAV & $2(16,7 \%)$ \\
\hline Dor & $1(8,3 \%)$ \\
\hline Cefaleia + dor em olho + lesão cutânea & $1(8,3 \%)$ \\
\hline Sinais de infecção & $1(8,3 \%)$ \\
\hline Dor + BAV + Oftalmoplegia + lesão cutânea & $1(8,3 \%)$ \\
\hline \multicolumn{2}{|l|}{ ABORDAGEM CIRÚRGICA n (\%) } \\
\hline Orbitotomia & $5(41,7 \%)$ \\
\hline Acesso por ferimento & $3(25 \%)$ \\
\hline Via endoscópica & $1(8,3 \%)$ \\
\hline Via endoscópica + transorbital combinada & $1(8,3 \%)$ \\
\hline Via endoscópica + orbitotomia infra-orbital & $1(8,3 \%)$ \\
\hline Intraocular & $1(8,3 \%)$ \\
\hline \multicolumn{2}{|l|}{ SEQUELAS n (\%) } \\
\hline Ausência de sequelas & $6(50 \%)$ \\
\hline Baixa acuidade visual & $3(25 \%)$ \\
\hline Ptose & $1(8,3 \%)$ \\
\hline Diplopia & $1(8,3 \%)$ \\
\hline Ausência de percepção luminosa & $1(8,3 \%)$ \\
\hline
\end{tabular}

Nota: 1- foram considerados sinais de infecção: febre, naúsea, vômito, palidez, sudorese e dispneia. 2- Lesões cutâneas se referem a: ferimentos corto-contusos e/ou fístulas cutâneas. Fonte: Autores. 


\section{Discussão}

Esta revisão confirmou que a ocorrência de infecção orbital por corpo estranho penetrante, decorrente de um acidente motociclístico e com severa limitação de abertura bucal, é um evento raro e pouco reportado na literatura. Além disso, os sinais clínicos mais frequentes associados a infecção orbital por corpo estranho são oftalmoplegia, abscesso, baixa acuidade visual, dor e lesão cutânea. É válido destacar que no nosso estudo, além do paciente apresentar baixa acuidade visual e oftalmoplegia, associada à infecção e proptose, ele apresentou uma severa limitação da abertura bucal, sintoma esse que ainda não foi relatado no âmbito de infecções orbitárias em decorrência de corpo estranho penetrante. O corpo estranho transfixava o assoalho da orbita e impactava o processo coronóide da mandíbula, limitando sua movimentação, e consequentemente, a abertura bucal. Além da impactação mecânica, o desenvolvimento de abscessos envolvendo os espaços ao redor dos músculos da mastigação são umas das fontes comuns de trismo (Luyk and Steinberg., 1990). Por esse motivo, mesmo após remoção de corpo estranho que causava uma impacção mecânica, limitando a abertura bucal, o paciente necessitou de fisioterapia coadjuvante para tratar trismo mandibular residual, causado pelo processo inflamatório/infeccioso.

A tomografia computadorizada (TC) é considerada padrão ouro para a identificação de corpos estranhos penetrantes orbitais, visto que, devido à vantagem de uma resolução geométrica mais alta, surge a oportunidade de distinguir os tecidos calculando suas unidades de Hounsfield (HU), sendo um método barato e rápido, apesar disso, pode ser usada também a ressonância magnética (Chen et al., 2015; Cho et al., 2017; Bayramoglu et al., 2018; Singh et al., 2018; Mirzaei et al., 2019; Szabo et al., 2019). Entretanto, o diagnóstico e o gerenciamento são desafiadores, porque essas técnicas de imagem têm limitações e às vezes são inconclusivas, especialmente para corpos estranhos de madeira (Chen et al., 2015). Uma das razões para esses desafios está relacionada ao grande número de variantes que contribuem para a aparência de corpos estranhos de madeira nas imagens, como tamanho e forma, tipo de madeira (macia ou dura), densidade física, estado de aeração, fresco, grau de absorção de água dos tecidos adjacentes e o tempo decorrido desde o início da lesão. A imagem formada por materiais de madeira é frequentemente confundida com gordura ou ar na TC, podendo contribuir para um diagnóstico confuso e equivocado (Jusué-Torres et al., 2016; Cho et al., 2017; Mirzaei et al., 2019; Szabo et al., 2019). Logo, detectar corpo estranho de madeira na imagem é difícil (Jusué-Torres et al., 2016), porque ela possui baixa densidade de Hounsfield deixando mais complicado distinguir do ar, simulando pneumocefalia e pneumo-orbita (Cho et al., 2017). Ao nosso caso, foi realizada a TC com a finalidade de localização exata do corpo estranho e limites das lesões para o estudo da possível remoção cirúrgica.

Como visto durante nosso estudo de revisão, a maioria dos casos são pacientes do sexo masculino, com idade média de 31, 4 anos e etiologia de acidente de trabalho. No presente relato de caso, o paciente inicialmente foi atendido em outro serviço, e por não apresentar lesões perceptíveis, o mesmo recebeu alta hospitalar. Por desenvolver um quadro de infeção orbital e ser atendido após dois meses no serviço de oftalmologia onde presumiram a possível existência de corpo estranho, foi encaminhado para o serviço de CTBMF, que através da anamnese com histórico de acidente motociclístico pregresso e exame de imagem (tomografia computadorizada) verificou o corpo estranho.

A estratégia terapêutica está diretamente relacionada com as características clínicas do paciente, o material e localização do corpo estranho e principalmente pela presença de infecção (Mirzaei et al., 2019; Szabo et al., 2019). Devido a órbita ser um espaço pequeno e com alta densidade de elementos funcionais, a remoção cirúrgica do corpo estranho intraorbital pode ser muito difícil e propensa a complicações visuais, sendo possível resultar na remoção incompleta (Szabo et al., 2019). Tal remoção se torna ainda mais complexa devido à anatomia piramidal da órbita e proximidade de estruturas críticas, como o conteúdo da fissura orbital superior, o nervo óptico, os vasos etmoidais e as estruturas cranianas (Moraes et al., 2015; Blackhall and Laraway, 2016; Tabibkhooei et al., 2019. A questão de saber se o corpo estranho deve ser removido permanece controverso (Szabo et al., 2019), corpos estranhos de madeira são frágeis, difíceis de remover completamente e podem fraturar na órbita, resultando em remoção incompleta, sendo assim, o procedimento cirúrgico é muito mais difícil em comparação com 
corpos estranhos de metal ( $\mathrm{Li}$ et al., 2016; Szabo et al., 2019). No nosso relato, em decorrência do quadro de infecção do paciente (leucocitose), com BAV e limitação da abertura bucal e dos movimentos oculares, ficou claro que a realização do procedimento cirúrgico era imprescindível para a vida do paciente.

As indicações para remoção cirúrgica de corpos estranhos incluem toxicidade, restrições mecânicas da mobilidade ocular, déficits neurológicos, compressão das estruturas intraorbitais, exoftalmia, desenvolvimento de infecção, formação de fístula orbital e dor. Corpos estranhos localizados na órbita anterior são geralmente removidos usando uma abordagem anterior e a remoção por essa abordagem apresenta sempre um risco de sangramento e mortalidade significativos causados pela remoção abrupta do efeito de tamponamento aplicado pelo corpo estranho à área lacerada (Singh et al., 2018; Mirzaei et al., 2019). A remoção cirúrgica foi realizada por acesso infraorbital para dispor de um acesso à órbita. A nossa revisão mostrou que a abordagem cirúrgica mais utilizada foi a orbitotomia (41,7\%), seguida da abordagem pela própria lesão (25\%), dessa maneira, a abordagem utilizada no nosso caso é concordante com a mais frequente, tendo em vista que o corpo estranho foi removido através de uma orbitotomia anterior.

Após a cirurgia de remoção, esta revisão apontou que a maioria dos pacientes (50\%) não apresentaram sequelas. Em contra partida, quando falamos em sequela, a mais recorrente foi a BAV, totalizando $25 \%$ dos casos estudados. No caso em questão, o paciente evoluiu com BAV no pós operatório, porém com melhora ao quadro inicial, e em acompanhamento ambulatorial com oftalmologia, aguardando cirurgia corretiva.

\section{Considerações Finais}

Por fim, o material do corpo estranho pode determinar o seu potencial risco infeccioso (Ghadersohi et al., 2017). Como vimos, a madeira possui um alto risco de causar infecção, assim, uma vez que definido o diagnóstico esses pacientes devem ser tratados imediatamente por uma equipe multidisciplinar, pois devido à anatomia da órbita e a proximidade com a região intracraniana, já abordada nesse estudo, à infecção deve ser tratada imediatamente. O exame de imagem é imprescindível para a condução do caso, afinal é através desse exame que se torna possível à verificação do material do corpo estranho (Unidades de Hounsfield) e se há impactação do processo coronóide mandibular.

Na literatura, estudos científicos relacionados a corpos estranhos em órbita são abundantes. Porém, essa efemeridade diretamente relacionada com o trismo, ainda detém de estudos escassos. Dessa forma, torna-se necessário a publicação de mais artigos em todos os ambitos de pesquisa acerca desse tema. A fim de produzir uma melhor contribuição acadêmica global.

\section{Agradecimentos}

Agradecemos especialmente ao oftalmologista Carlos A. T. de Araújo por sua colaboração com o atendimento ao paciente.

\section{Referências}

Bayramoğlu, S. E., Sayın, N., Erdogan, M., Yıldız Ekinci, D., Uzunlulu, N., \& Bayramoglu, Z. (2018). Diagnóstico tardio de corpo estranho intraorbital de madeira. Orbit, 37 (6), 468-471.

Bilge, A. D., Yılmaz, H., \& Naqadan, F. (2016). İntraorbital yabancı cisimler: Klinik özellikleri ve cerrahi çıkartım sonuçları. Ulusal Travma ve Acil Cerrahi Dergisi, 22(5), 432-436.

Blackhall, K. K., \& Laraway, D. C. (2016). Penetrating retro-orbital foreign body-large glass shards: A maxillofacial surgery case report. SAGE open medical case reports, 4, 2050313X15622890.

Chen, J., Shen, T., Wu, Y., \& Yan, J. (2015). Clinical characteristics and surgical treatment of intraorbital foreign bodies in a tertiary eye center. Journal of Craniofacial Surgery, 26(6), e486-e489. 
Research, Society and Development, v. 10, n. 4, e32310414280, 2021

(CC BY 4.0) | ISSN 2525-3409 | DOI: http://dx.doi.org/10.33448/rsd-v10i4.14280

Cho, W. K., Ko, A. C., Eatamadi, H., Al-Ali, A., Abboud, J. P., Kikkawa, D. O., \& Korn, B. S. (2017). Orbital and orbitocranial trauma from pencil fragments: role of timely diagnosis and management. American journal of ophthalmology, 180, 46-54.

Czyz, C. N., Petrie, T. P., Harder, J. D., Cahill, K. V., \& Foster, J. A. (2012). Intraorbital foreign body projectile as a consideration for unilateral pupillary defect. International journal of emergency medicine, 5(1), 1-4.

Ghadersohi, S., Ference, E. H., Detwiller, K., \& Kern, R. C. (2017). Presentation, workup, and management of penetrating transorbital and transnasal injuries: A case report and systematic review. American Journal of Rhinology \& Allergy, 31(2), e29-e34.

Jusué-Torres, I., Burks, S. S., Levine, C. G., Bhatia, R. G., Casiano, R., \& Bullock, R. (2016). Wooden foreign body in the skull base: how did we miss it?. World neurosurgery, 92, 580-e5.

Kang, S. J., \& Jeon, S. P. (2012). Surgical treatment of periorbital foreign body. Journal of Craniofacial Surgery, 23(6), e603-e605.

Kim, U. R., \& Sivaraman, K. R. (2013). Penetrating orbital injuries from plant material during pond and river diving. Indian journal of ophthalmology, 61(2), 76.

Li, J., Zhou, L. P., Jin, J., \& Yuan, H. F. (2016). Clinical diagnosis and treatment of intraorbital wooden foreign bodies. Chinese Journal of Traumatology, 19(6), 322-325.

Luyk, N. H., \& Steinberg, B. (1990). Aetiology and diagnosis of clinically evident jaw trismus. Australian dental journal, 35(6), 523-529.

Miller, K. E., \& Coan, E. B. (2016). Penetrating orbital injury from a needlefish. Military medicine, 181(8), e962-e964.

Moretti, A., Laus, M., Crescenzi, D., \& Croce, A. (2012). Corpo estranho periorbital: relato de caso. Journal of Medical case reports, 6 (1), 91.

Mirzaei, F., Salehpour, F., Shokuhi, G., Kermani, T. A., Salehi, S., \& Parsay, S. (2019). An unusual case of intra orbital foreign body; diagnosis, management, and outcome: a case report. BMC surgery, 19(1), 76.

Mohapatra, SSD, Das, J., \& Bhattacharjee, H. (2020). Um caso incomum de corpo estranho orbitocranial de madeira com resultado surpreendente: relato de caso. Jornal indiano de oftalmologia , 68 (1), 219.

Morais, HHA, Barbalho, JCM, de Souza Dias, T., Grempel, R., \& de Holanda Vasconcellos, RJ (2015). Abordagem temporal para remoção de um grande corpo estranho orbital. Trauma e reconstrução craniomaxilofacial , 8 (3), 234-238.

Oliveira, EM, de Arruda, JAA, Nascimento, PAM, Neiva, IM, Mesquita, RA, \& Souza, LN (2018). Uma atualização sobre o tratamento da osteomielite em um paciente com picnodisostose. Journal of Oral and Maxillofacial Surgery, 76 (10), 2136-e1.

Rajendran, S., \& Rikhotso, R. E. (2019). A fully retained knife blade within the face-An unusual sight. Oral and Maxillofacial Surgery Cases, 5(1), 100080.

Scofield-Kaplan, S. M., Weidman, E. K., Moonis, G., \& Glass, L. R. D. (2019). Corpo estranho orbital de madeira manifestando-se como hiperdensidade na tomografia computadorizada. Journal of American Association for Pediatric Ophthalmology and Strabismus, 23 (1), 45-47.

Singh, A., Sadique, S. I., \& Ghosh, S. N. (2018). Intraorbital foreign body with intracranial extension: a case series. International Clinical Neuroscience Journal, 5(1), 43 .

Szabo, B., Pascalau, R., Bartoè, D., Bartos, A., \& Szabo, I. (2019). Intraorbital penetrating and retained foreign bodies-a neurosurgical case series. Turk Neurosurg, 29(4), 538-548.

Swathi, N., \& Umadevi, J. (2014). An undetected intraorbital foreign body after a "trivial” facial injury. Journal of Craniofacial Surgery, 25(5), $1782-1783$.

Tabibkhooei, A., Aslaninia, A., \& Anousha, K. (2019). Lesão Penetrante na Base do Crânio Transorbital Infantil: Relato de 2 Casos e Revisão da Literatura. World neururgery, $131,213-216$.

Topazian, R. G., Goldberg, M. H., \& Rupp, J. R. (2006). Infecções orais e maxilofaciais. Santos.

Whittemore, R., \& Knafl, K. (2005). The integrative review: updated methodology. Journal of advanced nursing, 52(5), 546-553.

Wladis, E. J., Pappa, C., \& Cavaliere, L. F. (2011). Anticyclic-citrullinated protein antibodies in the diagnosis of ophthalmic inflammatory disease. Ophthalmic Plastic \& Reconstructive Surgery, 27(1), e1-e2. 